\title{
From the CSIRO
}

\section{Bat super immunity to lethal disease could help protect people}

For the first time, researchers have uncovered a unique ability in bats which allows them to carry but remain unaffected by lethal diseases. Bats are a natural host for more than 100 viruses, some of which are lethal to people, including Middle East respiratory syndrome coronavirus, Ebola virus and Hendra virus; however, bats do not get sick or show signs of disease from these viruses.

Published in the journal Proceedings of the National Academy of Sciences, the new research examines the genes and immune system of the Australian black flying fox, with surprising results (doi: 10.1073/pnas 1518240113).

"We focused on innate immunity of bats, in particular the role of interferons - which are integral for innate immune responses in mammals - to understand what's special about how bats respond to invading viruses," leading CSIRO bat immunologist Dr Michelle Baker said.

"Interestingly, we have shown that bats only have three interferon $\alpha$ genes, which is about a quarter of the number of interferon $\alpha$ genes we find in people.

"This is surprising given bats have this unique ability to control viral infections that are lethal in people and yet they can do this with a lower number of interferons."
The research showed that bats express a heightened innate immune response even when they were not infected with any detectable virus.

"Unlike people and mice, who activate their immune systems only in response to infection, bats' interferon $\alpha$ is constantly 'switched on', acting as a $24 / 7$ frontline defence against diseases," Dr Baker said.

"If we can redirect other species' immune responses to behave in a similar manner to that of bats, then the high death rate associated with diseases, such as Ebola, could be a thing of the past."

Led by the CSIRO, this international research effort included expertise from the CSIRO, Duke-NUS Medical School and the Burnet Institute.

Australian Animal Health Laboratory, Health and Biosecurity Business Unit, CSIRO

doi: 10.5694/mja16.00346

Podcast with Dr Michelle Baker available at www.mja.com.au/multimedia/podcasts 